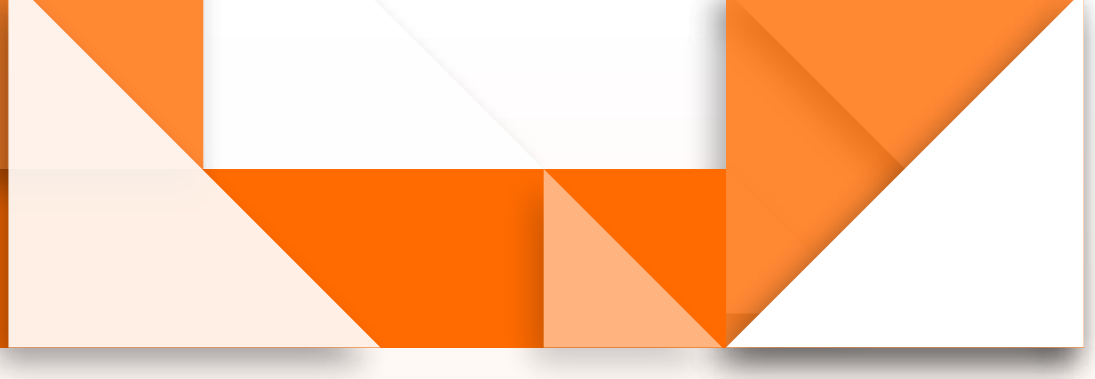

\title{
1) Un juego como estrategia de educación ambiental sobre la biodiversidad de Colombia
}

\section{- A Game as an Environmental Education Strategy on Colombia's Biodiversity}

- Um jogo como estratégia de educação ambiental sobre a biodiversidade da Colômbia

\section{Gilbert Salas López*}

\section{Resumen}

Debido a que el afecto por la fauna nativa desempeña un papel importante en su conservación y protección, este estudio cuasiexperimental con un diseño pre-/post- evaluó el efecto de un juego educativo sobre la identificación y el afecto por la fauna nativa de Colombia en 291 estudiantes de cuarto a noveno grado de 11 colegios públicos rurales y urbanos de Bogotá y Cundinamarca, quienes respondieron cuestionarios antes y después de 4-5 semanas de juego ad libitum durante el recreo escolar. Al contrastar el prejuego con el posjuego se encontró un aumento significativo en los porcentajes de identificación acertada de la fauna no nativa. Cuando se pidió a los estudiantes elegir sus cinco animales favoritos de un álbum, prejuego y posjuego eligieron más animales no nativos que nativos. Respecto a la elección de tres animales para ser salvados, los animales nativos y no nativos pre-/pos-juego fueron los mismos, con dos nativos y tres no nativos. No obstante, el porcentaje de alumnos que eligieron tres animales nativos para salvar tuvo un aumento de dos a cinco veces en el posjuego frente al prejuego. Aunque el resultado fue positivo respecto a la identificación de las especies no nativas, no se observó un cambio significativo en el afecto por los animales nativos, lo cual recalca el desafío y la necesidad de diseñar herramientas de educación ambiental que generen cambios profundos y duraderos en el afecto por la fauna nativa.

Palabras clave

educación ambiental; biodiversidad; fauna nativa
Biólogo, Magíster en Educación Ambiental; investigador Grupo Red Universidad y Ambiente GRUA.

gilbert.e.salas@gmail.com. ORCID: hHtp://0000-0002-3453-1104

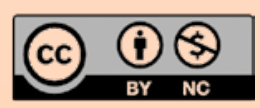




\section{Abstract}

Since the affection for native fauna plays an important role in its conservation and protection, this quasi-experimental study with a pre-/post- design evaluated the effect of an educational game on the identification and affection for the native fauna of Colombia in 291 fourth- to ninth-grade students from 11 rural and urban public schools in Bogotá and Cundinamarca who answered questionnaires before and after 4-5 weeks of ad libitum play during school recess. After contrasting pregame vs. postgame, we found a significant increase in the percentages of successful identification of non-native fauna. When asked to pick their top five favorite animals from an album before and after the game, students chose more non-native than natives animals. As for choosing three animals to be saved, the native and non-native animals before and after the game were the same, two of them native and three non-native. However, the percentage of students who chose three native animals to be saved increased two to five times after the game compared to before the game. Although the result was positive regarding the identification of non-native species, no significant change in the affection for native animals was observed, which emphasizes the challenge and the need to design environmental education tools that generate profound and lasting changes in the affection for native fauna.

\section{Keywords}

environmental education; biodiversity; native fauna

\section{Resumo}

Dado que o aprecio pela fauna nativa desempenha um papel importante em sua conservação e proteção, este estudo quase-experimental com um planejamento pré-/pós- avaliou o efeito do jogo educativo sobre a identificação e o afeto pela fauna nativa da Colômbia em 291 estudantes do quarto ao nono ano de onze colégios estaduais, rurais e urbanos de Bogotá e Cundinamarca, que responderam questionários antes de depois de 4-5 semanas de jogo ad libitum durante o recesso escolar. Ao contrastar o pré-jogo com o pós-jogo evidenciamos um incremento significativo nas porcentagens de identificação acertada da fauna não nativa. Quando solicitamos aos estudantes escolher seus cinco animais favoritos em um álbum, pré-jogo e pós-jogo, eles escolheram mais animais não nativos do que nativos. Em relação com a escolha de três animais para serem protegidos, os animais nativos e não nativos pré/pós-jogo foram os mesmos, com dois nativos e três não nativos. Contudo, a porcentagem de alunos que escolheram três animais nativos para serem protegidos cresceu de duas a cinco vezes no pós-jogo frente ao pré-jogo. Ainda que o resultado foi positivo em relação com a identificação das espécies não nativas, não observamos uma mudança significativa no afeto pelos animais nativos, o que destaca o desafio e a necessidade de criar ferramentas de educação ambiental que possam gerar mudanças profundas e duráveis no aprecio pela fauna nativa.

Palavras-chave

educação ambiental; biodiversidade; fauna nativa 


\section{Introducción}

La sexta extinción en masa está en curso (Leakey y Lewin, 1995, citados en Moyer-Horner, Kirby y Vaughan, 2010), y es la única ocasionada principalmente por la actividad humana (Moyer-Horner et al., 2010). Como evidencia de esta pérdida, la abundancia de las 3706 especies de vertebrados monitoreadas para el Índice Planeta Vivo disminuyó un promedio de $58 \%$ entre 1970 y 2012, y se estima que de no detenerse o revertirse esta tendencia, es posible que para 2020 las poblaciones de estas especies hayan disminuido un $67 \%$ respecto a 1970 (Fondo Mundial para la Naturaleza, 2016). Asimismo, en la Lista Roja de Especies Amenazadas de la UICN se encuentran más de 19000 especies de todo el mundo (Convention on Biological Diversity, 2013). Pero esta pérdida de especies, además de representar una amenaza para la existencia de los seres humanos, también es el único problema ambiental con el potencial de ser irreversible, y sus efectos podrían poner en peligro los medios de subsistencia de los que dependen las sociedades y las economías (Fondo para el Medio Ambiente Mundial, 2009). En otras palabras, por un lado la sociedad depende de la diversidad biológica para su subsistencia, y por otro la conservación de la biodiversidad depende de que la sociedad cambie sus expectativas, aspiraciones, conductas y uso de los recursos (Thiaw y Munang, 2012).

En cuanto a los resultados de las intervenciones emprendidas en el mundo para detener la extinción de especies, según la Secretaría del Convenio sobre la Diversidad Biológica (2010), ninguno de los países firmantes en la Cumbre Mundial sobre el Desarrollo Sostenible de 2002 en Johannesburgo logró una reducción importante en la tendencia hacia una pérdida de la biodiversidad, y se considera que uno de los obstáculos para lograr los objetivos establecidos en el Convenio es la falta de conocimiento y educación del público (Navarro-Pérez y Tidball, 2012). De acuerdo con estos antecedentes, y a la luz de las tendencias actuales, si no se presentan cambios a gran escala y a corto plazo, será imposible cumplir con varias de las metas de biodiversidad de Aichi, como la meta 1, la cual establece que para 2020, a lo sumo, las personas conocerán los valores de la biodiversidad, al igual que los mecanismos para conservarla y hacer uso de ella de manera sostenible, y la meta 12, que establece que para el 2020 se habrá prevenido la extinción de las especies amenazadas, y su estado de conservación habrá mejorado o se habrá mantenido sostenible (Convention on Biological Diversity, 2013).

Por otra parte, las personas solo se preocupan por lo que conocen (Balmford, Clegg, Coulson y Taylor, 2002). Por consiguiente, es prioritario que el público conozca la diversidad biológica que se encuentra a su alrededor, en particular en un país megadiverso como Colombia. Sin embargo, en este punto vale la pena recalcar que las estrategias de educación ambiental que utilizan especies icónicas del país, como el oso de anteojos o el jaguar, entre otros, sesgan la atención hacia animales poco vistos con una escasa interacción con los humanos y dejan en el olvido a miles de especies que requieren reconocimiento, como las lapas (Cuniculus paca), las arañas saltarinas (Salticidae), los pececillos de plata (Lespisma sp.), etc. De hecho, el tejido entre los animales y los humanos se pierde día a día, lo cual conduce irremediablemente a la desaparición de la biodiversidad, que es considerada por Taratsa (2010) como la base invisible de la existencia humana.

Pero este desequilibrio entre la abundancia del taxón y su conocimiento por el público no solo impacta la esfera cognitiva, sino también la afectiva. En particular, los niños 
tienen la tendencia a proteger las especies no nativas en lugar de las nativas (Ballouard, Brischoux y Bonnet, 201 1), y al ser la niñez el momento ideal para implementar la educación ambiental, la inclusión de diferentes taxones nativos en herramientas lúdicas podría generar excelentes resultados en la educación y motivación respecto al conocimiento y conservación de la diversidad biológica.

De manera ideal, la educación ambiental sobre biodiversidad, en particular la que se enfoca en la fauna nativa, debe incluir no solo herramientas lúdicas que puedan conectar la motivación, el afecto y el conocimiento, sino también especies de todos los taxones, como los mamíferos grandes y las especies pequeñas y menos conocidas que representen de una manera más realista la riqueza biológica del entorno. Asimismo, es importante que tales estrategias educativas aborden el tema de las especies nativas frente a las no nativas, y de esta manera establecer una relación de parentesco o familiaridad con las especies propias. Esto ayudaría a cumplir con la meta 9 de biodiversidad de Aichi, que establece que para el 2020 las especies invasoras estarán identificadas y priorizadas, y las especies más relevantes serán controladas o erradicadas (Convention on Biological Diversity, 2013).

En cuanto a la ubicación de esta investigación dentro de las corrientes de educación ambiental, debido a su enfoque educativo cognitivo y afectivo, y al uso del potencial pedagógico del juego, esta se ubica en la corriente naturalista (Sauvé, 2004). Por último, al considerar que el juego es una herramienta ideal para la educación ambiental (Taylor, 1983) que puede generar cambios en la actitud y la conducta de los niños (Salas, Rodríguez, \& Rivera, 2017), que los niños adoran los juegos (Prensky, 2006, citado en Bevilacqua, Ciarapica, Mazzuto y Paciarotti, 2015), y que las estrategias de educación sobre la fauna suelen utilizar únicamente las especies carismáticas, lo cual genera una discrepancia entre la biodiversidad existente y el conocimiento de los estudiantes sobre ella (Hodges, 2016). En este estudio se evaluó el efecto generado por un juego con especies nativas de diferentes taxones respecto a la identificación y el afecto frente a la fauna nativa de Colombia.

\section{Métodos}

\section{Diseño del estudio}

Este estudio comprendió tres fases:

- Fase de planeación y diseño: una vez se obtuvo el consentimiento informado de los padres y las instituciones, los docentes participantes (las personas a cargo de la investigación en cada institución) recibieron una sesión de capacitación sobre el uso del juego en escenarios educativos.

- Fase empírica: se aplicó una evaluación pre-juego y luego los estudiantes tuvieron una primera sesión de juego orientada de $\sim 1$ hora. 
Durante 4-5 semanas los estudiantes jugaron ad libitum en los recreos de la escuela, sin intervenciones didácticas relacionadas entre las sesiones de juego. Finalmente se aplicó una evaluación posjuego.

- Fase analítica: se recabaron las evaluaciones prejuego y posjuego y se tabularon y analizaron los datos.

La población de estudio estuvo compuesta por 291 estudiantes de grados cuarto a noveno de colegios públicos rurales y urbanos de Bogotá y Cundinamarca (véase la tabla 1).

Tabla 1. Datos demográficos de la población del estudio por tipo de colegio

\begin{tabular}{|l|l|}
\hline \multicolumn{1}{|c|}{ Colegios rurales } & \multicolumn{1}{c|}{ Colegios urbanos } \\
\hline $\mathrm{n}=143$ & $\mathrm{n}=148$ \\
\hline $\begin{array}{l}\text { Edad promedio }=12,4 \\
\text { años }\end{array}$ & $\begin{array}{l}\text { Edad promedio }=11,8 \\
\text { años }\end{array}$ \\
\hline $\begin{array}{l}\text { Rango de edad }=11-18 \\
\text { años }\end{array}$ & $\begin{array}{l}\text { Rango de edad }=9-17 \\
\text { años }\end{array}$ \\
\hline
\end{tabular}

Fuente: elaboración propia.

La evaluación contó con un álbum de fotografías $(5 \times 4 \mathrm{~cm})$ numeradas de 72 animales nativos y no nativos de Colombia (véase la figura 1) y con un cuestionario de tres preguntas: (1) Escribe el número correspondiente a los animales del álbum que no son de Colombia; (2) Escribe el número correspondiente a tus cinco animales favoritos del álbum; (3) si tuvieras que salvar a tres de los animales en la siguiente tabla, ¿̇a cuáles salvarías? Los animales incluidos en la tabla mencionada fueron: jaguar (Panthera onca), elefante, panda, cocodrilo del Orinoco (Crocodylus intermedius), danta (Tapirus terrestris), rinoceronte, cóndor (Vultur gryphus), koala, rana dardo dorada (Phyllobates terribilis), oso de anteojos (Tremarctos ornatus), tigre y león. Aunque las preguntas prejuego y posjuego fueron las mismas, su secuencia fue diferente, lo cual se ha utilizado en algunas mediciones de la efectividad del aprendizaje basado en juegos (All, Nuñez Castellar y Looy, 2014).

Se eligió un juego de cartas por ser un recurso conocido en la educación, cuyo uso para mejorar el aprendizaje se ha estudiado ampliamente (Turkay, Adinolf y Tirthali, 2012). En este caso, la dinámica del juego se basa en la información científica y taxonómica para simular las posibles relaciones tróficas de diferentes grupos de animales (insectos, arácnidos, anfibios, reptiles, aves y mamíferos). Cada carta contiene la fotografía de un animal, el nombre científico, el nombre común en inglés y español, y un octágono con información del tamaño en centímetros y la dieta del animal. Los ecosistemas y los animales representados en las cartas se encuentran en Colombia. De acuerdo con lo anterior, se esperaba que la mecánica del juego (véase la figura 2) no solo motivara la tendencia innata del humano a enfocarse en la vida y en los procesos de vida, tal como propone la hipótesis de biofilia de Edward O. Wilson (Johnson, 1994), sino también la gran capacidad que tienen los niños de aprender sobre las criaturas, artificiales o naturales, como puede evidenciarse por el hecho de que niños de 8 años fueron capaces de identificar casi el $80 \%$ de una muestra extraída de 150 especies "artificiales" de Pokémon (Balmford Clegg, Coulson y Taylor, 2002). Asimismo, se esperaba que la visualización de los animales durante el juego pudiera llevar a una identificación en la naturaleza, la cual es muy similar a la identificación a partir de fotografías (Palmberg et al., 2015). 


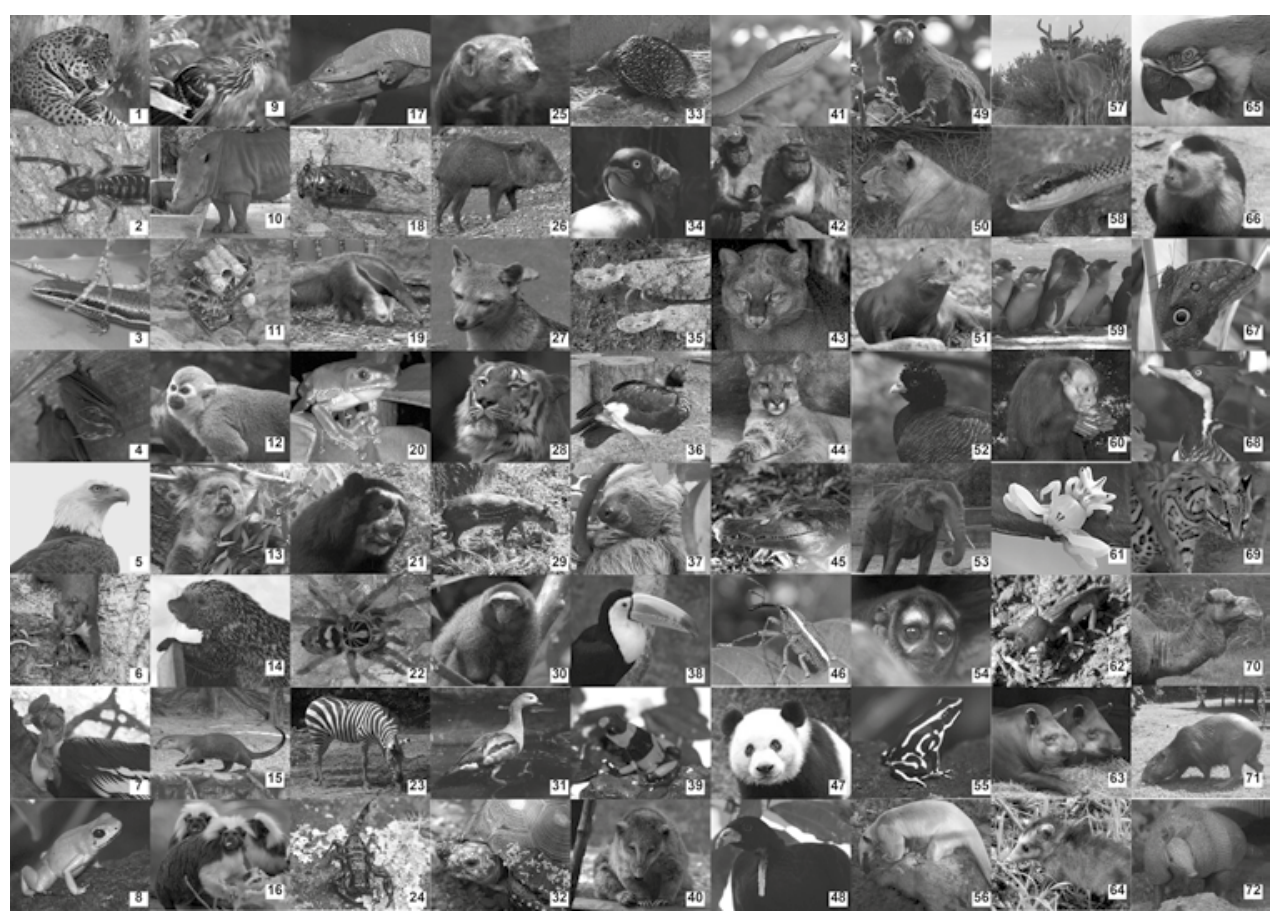

Figura 1. Álbum de animales no nativos y nativos de Colombia empleado para la evaluación

Fuente: elaboración propia.
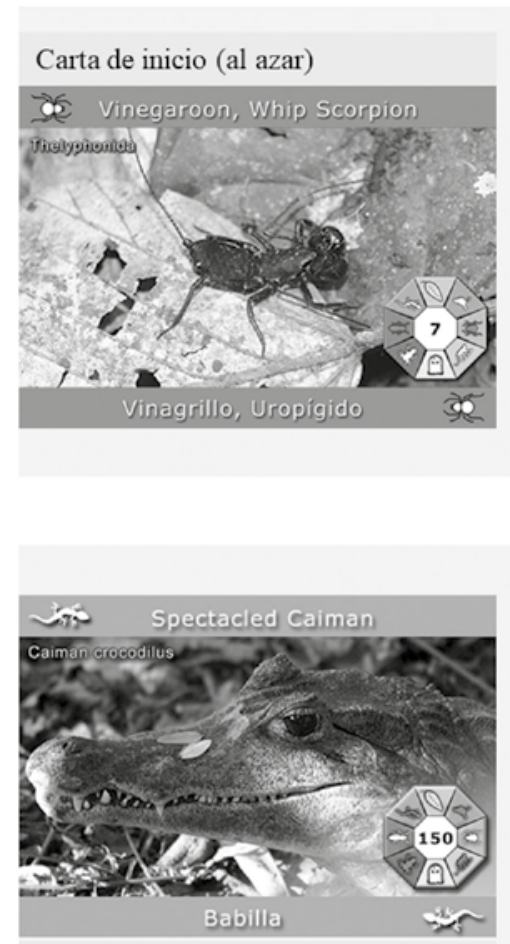

El siguiente jugador debe lanzar una carta con un animal que coma

reptiles que tenga un tamaño $>150$ $\mathrm{cm}$... Y así sucesivamente.
El jugador debe lanzar la carta de un animal que coma arácnidos y que tenga un tamaño $>7$.

El jugador debe lanzar la carta de un animal que coma reptiles y que tenga un tamaño $>140$.

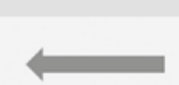

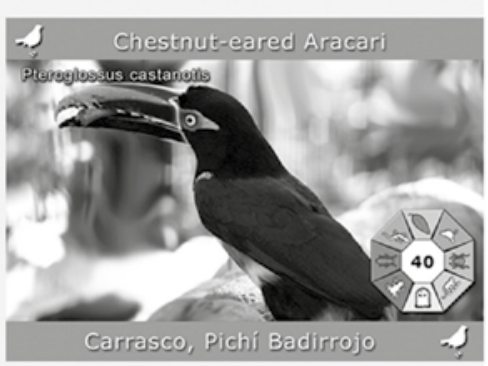

El jugador debe lanzar la carta de un animal que coma aves y que tenga un tamaño $>40$.

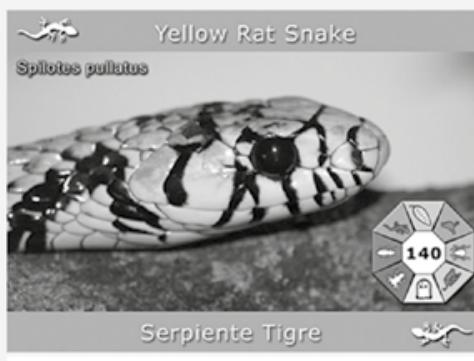

Convenciones: Animal de $180 \mathrm{~cm}$, que come anfibios, reptiles, aves y mamíferos.

Figura 2. Dinámica del juego

Fuente: elaboración propia. 


\section{Resultados}

\section{Reconocimiento de los animales no nativos}

La media general (urbanos + rurales) del puntaje de acierto en la identificación de los animales no nativos presentes en el álbum fue de 0,35 $\pm 0,23$ prejuego y de 0,41 $\pm 0,23$ posjuego. Cuando se comparó la media general del puntaje de aciertos en la identificación de los animales no nativos presentes en el álbum prejuego frente a posjuego se encontró una diferencia estadísticamente significativa (prueba $t$ para urbanos + rurales: $t=-4,74$; $p<0,01)$. Por su parte, en los colegios rurales la media del puntaje de aciertos en la identificación de los animales no nativos presentes en el álbum fue de 0,36 $\pm 0,22$ prejuego y de $0,40 \pm 0,24$ posjuego, y en los colegios urbanos fue de 0,34 $\pm 0,23$ prejuego y de $0,41 \pm 0,23$ posjuego. Asimismo, cuando se comparó la media del puntaje de aciertos en la identificación de los animales no nativos presentes en el álbum prejuego versus posjuego en los colegios urbanos y rurales por separado se encontraron diferencias estadísticamente significativas (prueba + para rurales prejuego frente a posjuego: $t=-2,48, p<0,05 \mid$ prueba t para urbanos prejuego frente a posjuego: $t=-4,066, p<0,01$ ) (véase la figura 3).

Los cinco animales que tuvieron mayor dificultad en ser identificados acertadamente como no nativos fueron el equidna, el águila calva, el tigre, el león y el koala, cuyo porcentaje de acierto más alto solo alcanzó el 31 \% posjuego en el caso del koala. Por otra parte, el porcentaje de acierto más alto prejuego fue del $57 \%$ para el pingüino y posjuego fue del $65 \%$ para el camello. En general, la identificación de los animales no nativos mejoró posjuego versus prejuego, excepto en el caso del pingüino (véase la figura 4).

Los animales nativos que con mayor frecuencia fueron considerados erróneamente como no nativos, tanto prejuego como posjuego, fueron: la araña cangrejo, la machaca, la pava hedionda y el venado cola blanca (véase la figura 5).

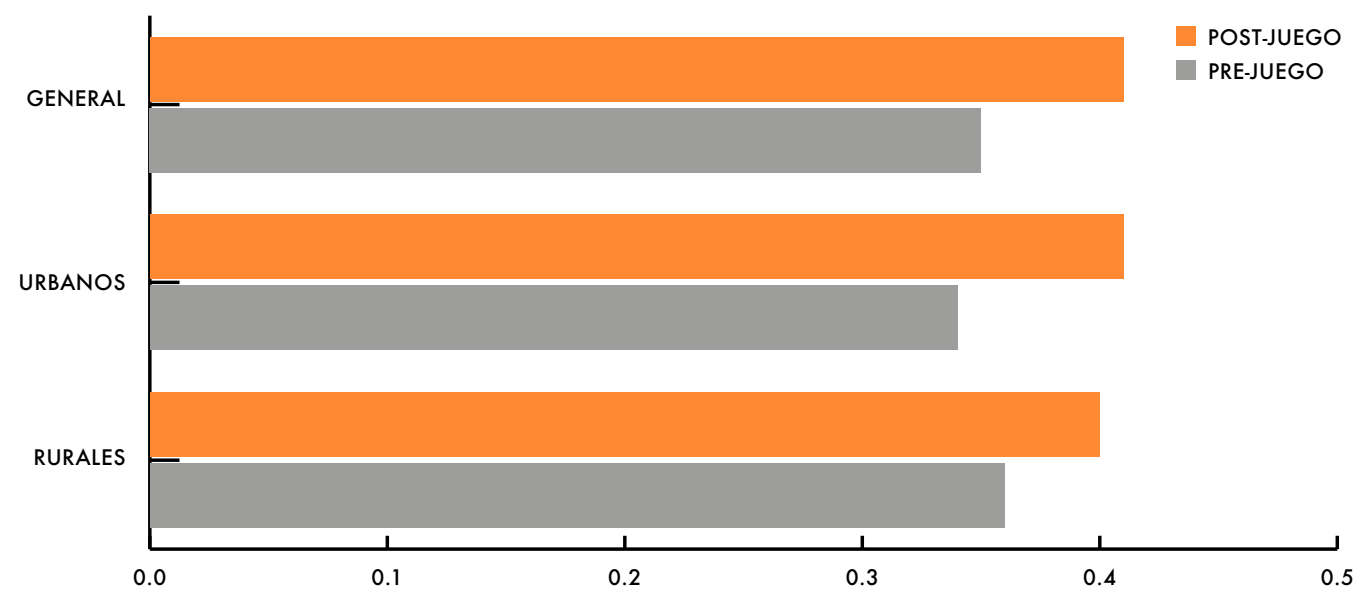

Figura 3. Puntaje de acierto en la identificación de los animales no nativos presentes en el álbum, prejuego y postjuego

Fuente: elaboración propia. 


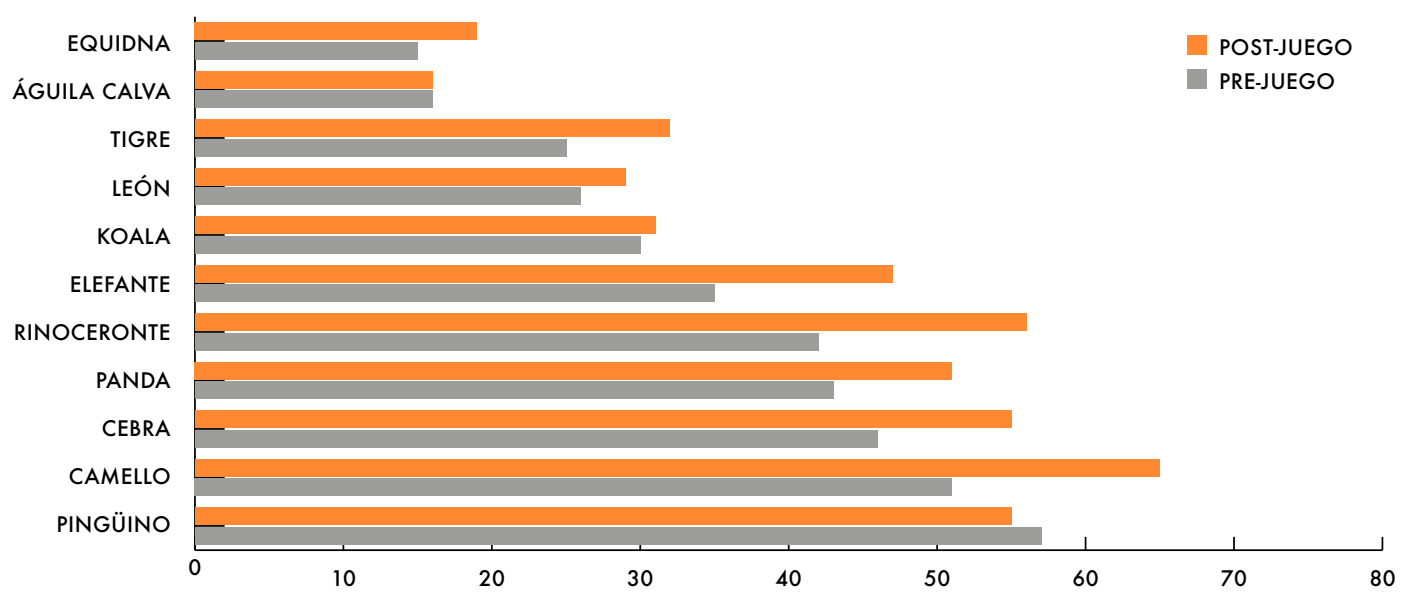

Figura 4. Porcentaje de acierto en la identificación de cada uno de los animales no nativos presentes en el álbum

Fuente: elaboración propia.
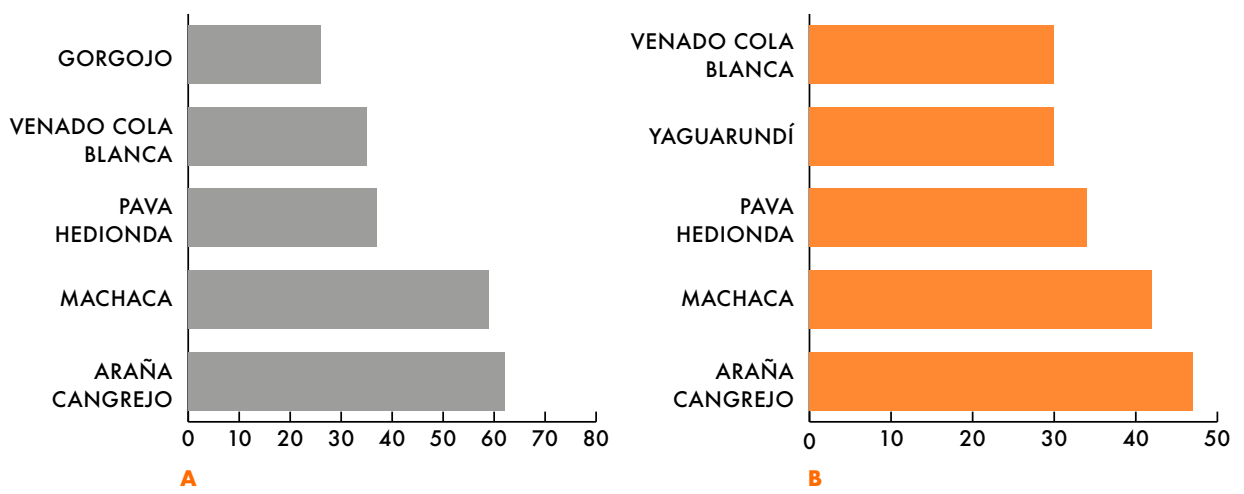

Figura 5. Cinco animales nativos que con mayor frecuencia fueron identificados erróneamente como no nativos (A) prejuego y (B) posjuego [araña cangrejo (Thomisidae); machaca (Fulgora sp.); pava hedionda (Opisthocomus hoazin); venado cola blanca (Odocoileus virginianus goudotii); gorgojo (Curculionidae); yaguarundí (Puma yagouaroundi)]

Fuente: elaboración propia.

\section{Elección de cinco animales favoritos del álbum}

Tanto en prejuego y posjuego los estudiantes de colegios rurales y urbanos escogieron los mismos cinco animales favoritos del álbum. En general, el jaguar fue elegido por el $60 \%$ de los estudiantes dentro de sus cinco animales favoritos del álbum y ocupó el primer lugar seguido por el panda (57\%), el tigre (50 \%), el águila calva (44 \%) y la guacamaya (27\%). Solo dos animales nativos estuvieron dentro de los cinco animales favoritos del álbum. Asimismo, aunque los animales nativos (61/72) obtuvieron el 55 \% (784/1430), la proporción de votos por 
animal fue muy baja (13) en comparación con los animales no nativos (59 votos por animal) (véase la figura 6).

\section{Preferencia por los animales de la fauna nativa frente a la no nativa}

No se observaron cambios en el patrón del número de animales nativos elegidos en la evaluación prejuego versus posjuego de los colegios rurales (número de animales nativos elegidos, de mayor a menor porcentaje: prejuego 1 | 2 | 0 | 3 vs. posjuego $1 \mid 2$ | 0 | 3), urbanos (número de animales nativos elegidos, de mayor a menor porcentaje: prejuego 1 | 0 | 2 | 3 vs. posjuego 1 | 0 | 2 | 3), o en general (número de animales nativos elegidos, de mayor a menor porcentaje: prejuego $1|0| 2$ | 3 vs. posjuego 1 | 0 | 2 | 3). Sin embargo, en comparación con los colegios urbanos, los rurales tuvieron un mayor porcentaje de preferencia por tres animales nativos prejuego (urbanos $2 \%$ vs. rurales $6 \%$ ) y por dos animales nativos prejuego y posjuego (urbanos: prejuego $=18 \%$ y posjuego $=15 \%$ vs. rurales: prejuego $=29 \%$ y posjuego $=24 \%$ ). Respecto al porcentaje de alumnos que eligieron solo animales nativos (3/3), se observó un aumento

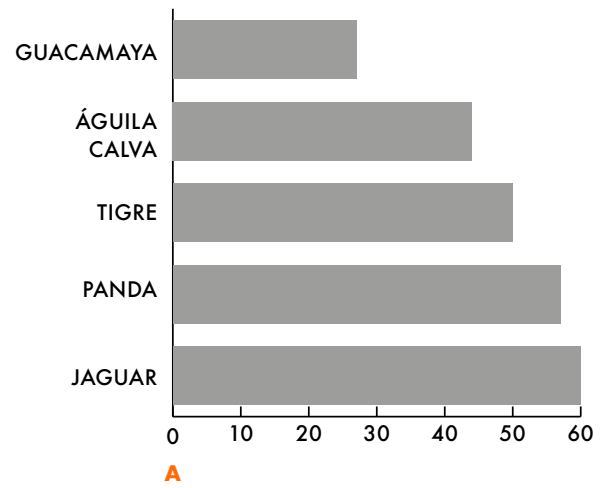

de dos a cinco veces en la evaluación prejuego vs. posjuego en los colegios rurales $(6 \%$ vs. $11 \%)$, urbanos (2\% vs. $11 \%$ ), y en general (4\% vs. $11 \%$ ). No obstante, el porcentaje de estudiantes que no escogieron animales nativos siempre fue superior al de los que escogieron tres animales nativos (véase la figura 7).

En cuanto a los animales elegidos para ser salvados, el panda tuvo los mayores porcentajes de votación en los colegios urbanos, rurales y en general, tanto en las evaluaciones prejuego como posjuego. Respecto a lo ocurrido en los colegios urbanos, los únicos animales nativos dentro de los cinco más votados prejuego fueron el oso de anteojos en el tercer puesto y el jaguar en el quinto puesto. Sin embargo, en la evaluación posjuego de los colegios urbanos el único animal nativo fue el oso de anteojos (tercer puesto). En los colegios rurales dos animales nativos estuvieron dentro de los cinco más votados: el oso de anteojos (tercer puesto prejuego y segundo puesto posjuego) y el cóndor (segundo puesto prejuego y cuarto puesto posjuego). En general, dos animales nativos estuvieron dentro de los cinco más votados: el oso de anteojos (segundo puesto prejuego y posjuego) y el cóndor (cuarto puesto prejuego y posjuego) (véase la figura 8).

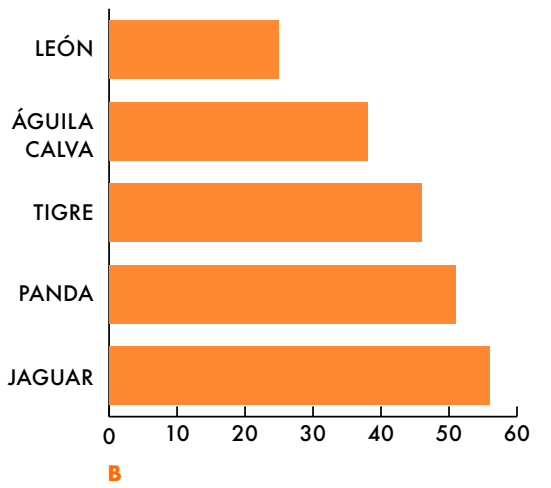

Figura 6. Cinco animales favoritos presentes en el álbum, en general (urbanos + rurales), (A) prejuego y (B) posjuego [guacamaya (Ara ararauna); jaguar (Panthera onca); panda (Ailuropoda melanoleuca); tigre (Panthera tigris); águila calva (Haliaeetus leucocephalus); león (Pantera leo)] 


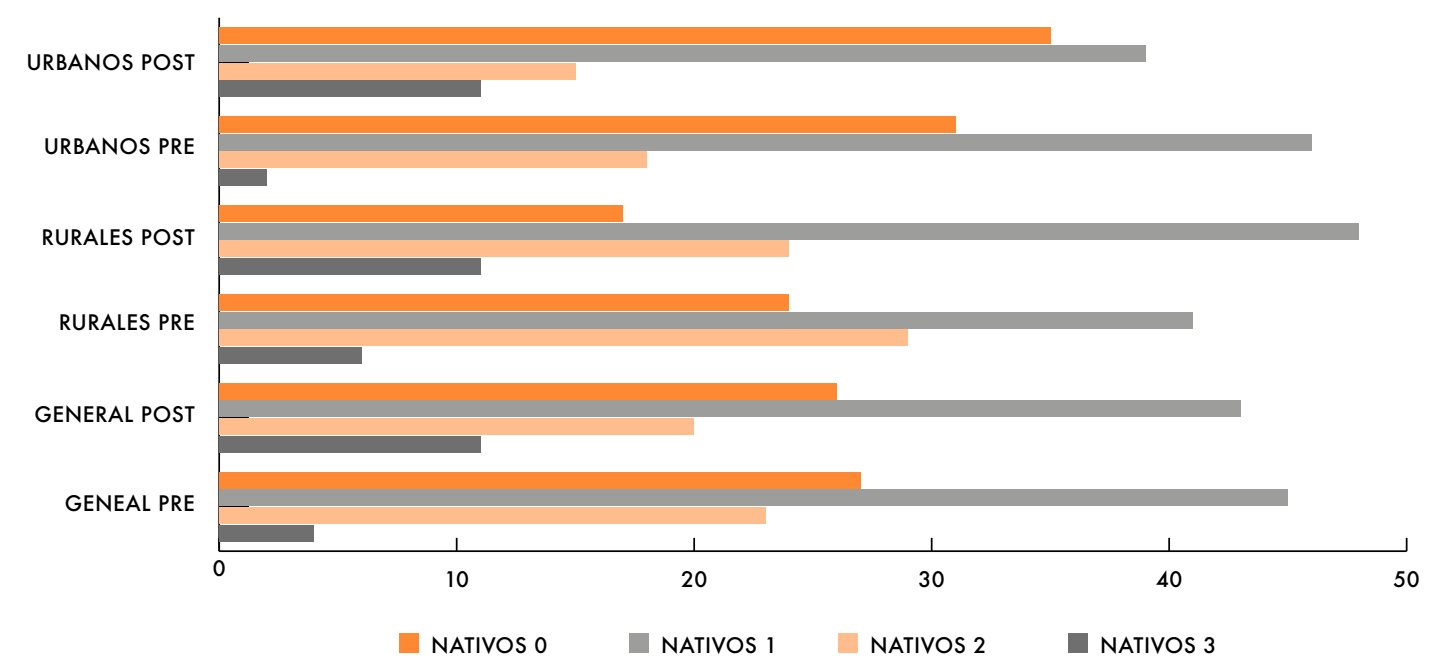

Figura 7. Porcentajes en que fueron elegidos ninguno, uno, dos o tres de los animales nativos propuestos en la pregunta, prejuego y posjuego, por tipo de colegio y en general Fuente: elaboración propia.
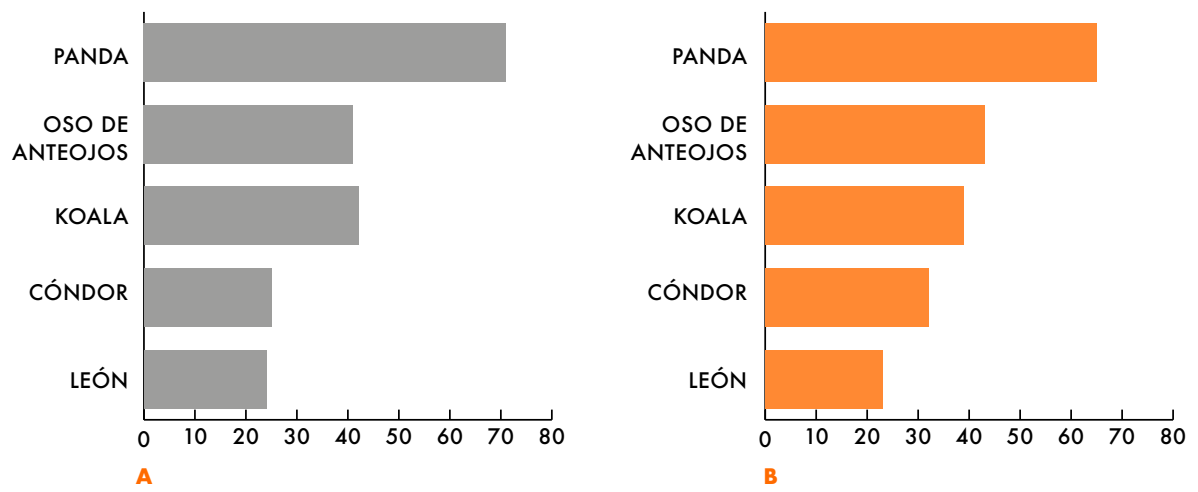

Figura 8. Porcentajes de votación por los diferentes animales de la fauna nativa y no nativa, en general (urbanos + rurales), (A) prejuego y (B) posjuego [león (Pantera leo); cóndor (Vultur gryphus); koala (Phascolarctos cinereus); oso de anteojos (Tremarctos ornatus); panda (Ailuropoda melanoleuca)]

Fuente: elaboración propia.

\section{Discusión}

\section{Identificación de los animales no nativos}

Aunque se encontró una diferencia estadísticamente significativa entre el puntaje promedio de acierto prejuego vs. posjuego con los colegios urbanos y rurales combinados (en general), el puntaje de acierto prejuego fue de solo $35 \%$ y posjuego de $41 \%$, algo que se relaciona con que en general los niños tienen un escaso conocimiento de los animales (Kellert, 1984; Randler, 2008). Asimismo, aunque el puntaje de acierto fue similar entre los colegios urbanos y rurales 
(rurales: $36 \%$ prejuego y $40 \%$ posjuego vs. urbanos: $34 \%$ y $41 \%$ ), la diferencia a favor de los rurales prejuego coincide con el resultado de otro estudio, según el cual los niños en las poblaciones rurales suelen tener un mayor conocimiento de los animales en comparación con los que viven en grandes ciudades (Kellert, 1984).

Por otro lado, se encontró que algunos animales nativos que deberían ser reconocidos fácilmente, ya sea por ser tema de un dicho popular, como la machaca (59\% prejuego | $42 \%$ posjuego), o por ser la insignia de una región, como la pava hedionda (37\% prejuego | $34 \%$ posjuego) en la Orinoquía, o el venado cola blanca (35\% prejuego | $30 \%$ postjuego) en el páramo, fueron considerados erróneamente como no nativos, lo cual no solo ratifica el escaso conocimiento de los niños respecto a la fauna nativa, sino también lo difícil que puede llegar a ser la identificación de los animales, en este caso en particular, solo con una fotografía. En este sentido, cuando se enseña a los estudiantes a identificar animales debe tenerse en cuenta que aprender sobre las especies es complicado, y es importante incluir la información de la historia natural o de la ecología del animal (Randler y Bogner, 2006). No obstante, respecto a la identificación de los animales por medio de fotografías, cabe destacar el trabajo de Kosslyn (citado en Otero, 1999), quien determinó que los niños pequeños dependen en gran medida de imágenes cuando acceden a la información en su memoria.

Asimismo, en la evaluación se les pedía a los estudiantes que identificaran y clasificaran 72 animales entre nativos y no nativos; es posible que la identificación y clasificación de este número de animales haya sobrepasado las capacidades de los estudiantes. De hecho, mientras que en un estudio realizado por Mayer (1991) se consideró que en los años de secundaria debía enseñarse a identificar cerca de 250 especies, que es un número considerable, cuando Randler y Bogner (2006) escogieron 14 especies diferentes de aves encontraron tasas bajas de retención después de 6-8 semanas (citados en Randler, 2008), así que finalmente se determinó que para una tarea de identificación de aves son suficientes 6 especies (Randler y Bogner, 2006). A este respecto cabe señalar que el álbum incluía, solo en aves, 11 especies.

\section{Animales favoritos del álbum y preferencia por la fauna nativa frente a la no nativa}

Prejuego y posjuego, los estudiantes de colegios urbanos y rurales eligieron los mismos cinco animales favoritos presentes en el álbum, con leves diferencias en los porcentajes, e incluyeron tres animales no nativos y dos nativos. Sin embargo, cabe mencionar que algunos de los estudiantes incluyeron en la respuesta (solo se pedía el número del animal) el nombre, y algunas veces el número 1 estuvo acompañado por el nombre "leopardo". Debido al gran parecido entre el jaguar y el leopardo, es posible que los estudiantes en realidad eligieran al leopardo y no al jaguar. Como consecuencia, en general, el único animal que realmente entró en la categoría nativo es la guacamaya, que ocupó el quinto lugar con el $27 \%$ de los votos en la evaluación prejuego. En la evaluación posjuego la guacamaya fue desplazada por el león, que obtuvo los votos del $25 \%$ de los estudiantes. Por consiguiente, en la evaluación posjuego el único animal nativo entre los cinco favoritos fue el jaguar, con la salvedad hecha

Por otra parte, en la evaluación prejuego hubo tres mamíferos y dos aves, mientras que en la evaluación posjuego hubo 4 mamíferos y un ave, una predominancia de los mamíferos que refleja no solo las preferencias de grandes 
y chicos por los animales de mayor tamaño (Metrick y Weitzman, 1998; Ward et al., 1998) sino también el escaso entendimiento de la composición y abundancia de los diferentes taxones en los ecosistemas. Esto genera un sesgo en el interés hacia los mamíferos, las aves y los reptiles en comparación con los otros taxones (Snaddon, Turner y Foster, 2008).

En cuanto a la proporción de votos por animal nativo o no nativo se encontró que los nativos solo tuvieron 13 votos, mientras que los no nativos obtuvieron 59. No obstante, es importante tener en cuenta que los 11 animales no nativos incluidos en el álbum eran especies carismáticas, mientras que solo unas pocas de las especies nativas lo eran. Asimismo, ya que parece que el público general no distingue a las especies por ser "nativas o no nativas" (Bednar-Friedl et al., 2009), y en general este aspecto tiene poca importancia para la gente (Fischer, Langers, Bednar-Friedl, Geamana y Skogen, 2011), es posible que el atributo "nativo o no nativo" del animal no se relacione con el componente afectivo.

Respecto a la elección de tres animales para ser salvados entre doce nativos (6) y no nativos (6), todos los animales gozaban de una amplia popularidad ya que habían sido objeto de campañas para su protección y conservación, eran íconos nacionales o especies carismáticas/bandera. El panda, el oso de anteojos y el koala fueron los tres animales que encabezaron los listados de animales para salvar, seguidos por el cóndor y finalmente el león, lo cual no solo confirma el impacto de las campañas de protección y conservación de las especies en peligro de extinción, sino también que los mamíferos son los animales preferidos, seguidos por las aves y los reptiles, lo cual podría relacionarse con el principio de similitud (Tisdell, Wilson y Swarna Nantha, 2006).

En este contexto, parece que el afecto por los animales no se relaciona con la pertenencia a un territorio, sino más bien con otros factores, como la estética o el taxón. Por ejemplo, según Roque De Pinho, Grilo, Boone, Galvin y Snodgrass (2014), la apreciación estética que el ser humano hace de las especies animales influencia las actitudes respecto a la conservación de tales especies, y parece que las percepciones de las especies "feas" pueden volverse más positivas por medio del contacto directo con ellas, así que recomiendan mejorar la familiaridad de las personas con las especies poco vistas. Asimismo, en un estudio sobre los animales que más despertaban el interés de los visitantes en un zoológico se encontró que el taxón era el predictor más importante respecto al interés del visitante, es decir, la mayoría estaba más interesada en los mamíferos, aunque el tamaño del animal, su mayor actividad, y el hecho de que fuera una de las especies bandera también tuvieron que ver con la preferencia del público (Moss y Esson, 2010). En otras palabras, cuando se trata del interés o el afecto por los animales, tanto el taxón como la estética del animal son más importantes que la pertenencia o no al mismo contexto ecológico/social/cultural de la persona. Por ende, es evidente por qué los estudiantes que participaron en esta investigación 
seguían eligiendo los animales más llamativos, sin importar si pertenecían o no a su país.

En la última década se ha producido un flujo acelerado de hallazgos en múltiples disciplinas que apoyan una visión del afecto como una forma compleja entrelazada con la cognición en la orientación de la conducta racional, la recuperación de la memoria, la toma de decisiones y la creatividad, entre otras (Picard et al., 2004). Por consiguiente, puede esperarse que el componente afectivo desempeñe un papel muy importante en la toma de decisiones respecto a la protección y la conservación de las especies. Además, si se considera que la valoración del público estadounidense de los animales se basa en la manera en que las especies se han considerado desde la perspectiva histórica, en su utilidad para los humanos, y en la reacción emocional con ellas (Fox, 1990, citado en Wiley-Driscoll, 1995), entonces el diseño de las estrategias y herramientas de educación ambiental sobre biodiversidad deben enfocarse no solo en tratar de reconocer la importancia de los animales en la historia y la cotidianidad, sino también en favorecer una respuesta emocional positiva frente a ellos.

\section{El aprendizaje basado en juegos como herramienta para dinamizar conocimientos y generar cambios afectivos}

Se encontró que el juego produjo un efecto positivo sobre el conocimiento, pero no sobre el afecto por los animales nativos. A este respecto, vale la pena tener en cuenta que la intervención comprendió un juego que solo utiliza información visual, sin la interacción con especímenes in vivo, lo cual hace que su efecto sobre el componente afectivo sea muy escaso o inexistente. De hecho, cuando se comparó el aprendizaje cognitivo y afectivo en niños de cuarto grado después de manipular especímenes vivos o preservados de diferentes animales se encontró que el aprendizaje cognitivo a corto y a largo plazo se presentó con ambos tipos de contacto, pero los aumentos en el aprendizaje afectivo (medidos como cambios de actitud) solo se lograron cuando los niños manipularon los especímenes vivos (Sherwood, Rallis y Stone, 1989), algo muy similar a lo que podría esperarse al ver las fotografías de los animales sin tener la posibilidad de un contacto con los especímenes vivos.

Por otro lado, en los niños, las percepciones de los animales evolucionan y presentan diferentes etapas. En el estudio realizado por Kellert y Westervelt (1983), las percepciones de los niños entre segundo y quinto grado se caracterizaron por un aumento dramático en las preocupaciones emocionales y el afecto general por los animales, y entre el quinto y el octavo grado se observó una gran expansión en su comprensión intelectual y cognitiva de los animales. De acuerdo con lo anterior, la población del presente estudio, conformada por estudiantes de los grados cuarto a noveno, podría explicar el efecto del juego sobre el componente cognitivo y no sobre el afectivo, que debería abordarse tempranamente en la edad escolar, entre el segundo y el quinto grado.

En cuanto al juego y su papel como herramienta para dinamizar conocimientos, quizás lo más importante sea la motivación y el interés que esta metodología despierta en la población infantil. De hecho, no puede olvidarse que los niños adoran los juegos (Prensky, 2006, citado en Bevilacqua et al., 2015), y ya que la motivación es fundamental para el proceso de aprendizaje (Müller, Appleton, Valverde y Reynolds, 2015), y solo se aprende lo que se ama (Mora, 2017), entonces, en teoría, no es sorprendente que el juego generara un aumento en el conocimiento de los animales 
nativos, incluso sin la presencia de un tutor que potenciara o reforzara el papel del juego.

Por el contrario, el efecto del juego sobre la esfera afectiva no es tan directo como en el caso de los conocimientos. Por ejemplo, un estudio encontró que, aunque los dueños de mascotas solían tener actitudes más positivas por los animales silvestres en comparación con las personas que no tenían mascotas, esta diferencia desaparecía cuando se comparaban las actitudes por los animales menos populares (Bjerke, Østdahl y Kleiven, 2003), lo cual sugiere que es muy difícil cambiar las actitudes frente a los animales menos populares (Prokop y Tunnicliffe, 2008). En otras palabras, los cambios en el afecto son mucho más difíciles y complejos que en los conocimientos. En este sentido, si bien los procesos cognitivos y afectivos están relacionados, son diferentes; de hecho, las reacciones iniciales de los humanos a los estímulos suelen ser afectivas, no cognitivas, y por consiguiente podemos gustar o sentir temor de algo antes de conocerlo de manera precisa, incluso antes de saber qué es (Zajonc, 1980). De esta manera puede explicarse por qué las personas desarrollan sentimientos de afecto por animales que nunca han visto o acariciado, y aunque su conocimiento indica que todos son valiosos e importantes, incluso los menos populares, pueden llegar a sentir repulsión por aquellos con los que no tienen un vínculo afectivo, como se vio reflejado en este estudio.

\section{Conclusiones}

El juego incluyó animales de diferentes taxones. Su dinámica se basó en las relaciones tróficas, y todos los animales contaban con características que les permitían ganar o perder frente a otros; esto con el objetivo de enfatizar el papel y la importancia que tienen todos los animales en un ecosistema, cualquiera sea su tamaño, apariencia o taxón. Si bien el conocimiento de la fauna nativa aumentó prejuego versus posjuego, tal efecto no se reflejó en un cambio en los animales favoritos ni en los que debían salvarse. Es decir, conocer al animal, al igual que su estatus de pertenencia a la fauna nativa, no significó quererlo o preferirlo. En otras palabras, un cambio en el componente cognitivo no se relaciona con un cambio en el componente afectivo.

Ya que es posible amar u odiar un animal sin conocerlo, al igual que es posible conocerlo sin amarlo, y con el fin de aprovechar al máximo el desarrollo innato de las percepciones de los niños para lograr el mayor impacto posible de las intervenciones educativas relacionadas con el conocimiento, el afecto y la preocupación por los animales, es importante que las estrategias de educación ambiental relacionadas con la fauna nativa hagan una segmentación de la población objetivo. Es decir, en la primera infancia y en los niños pequeños es primordial que las estrategias de educación ambiental aborden la esfera afectiva; luego, cerca del paso de la primaria al bachillerato, deben hacer hincapié en el aprendizaje de datos y hechos, y a partir de la mitad del bachillerato deben 
abordar las relaciones éticas con los animales y los conceptos de ecología.

Aunque la metodología del estudio establecía el uso del juego durante el recreo sin el apoyo de un tutor, y este tuvo que competir por el tiempo de los niños contra los deportes y otras actividades, los resultados fueron prometedores. En efecto, de acuerdo con los resultados, el juego funcionó como un medio efectivo para indagar y dinamizar conocimientos entre pares, y más allá de los efectos sobre el conocimiento y el afecto, los docentes participantes mencionaron que sus estudiantes mostraron un aumento en la autonomía, el liderazgo, el trabajo en equipo, la equidad y la colaboración.

Por otra parte, el afecto por la fauna entre los niños y jóvenes, cualquiera sea su contexto, urbano o rural, no pareció relacionarse con que los animales sean nativos o no, sino más bien con la apariencia y el taxón, lo cual genera un sesgo en el conocimiento/afecto por los mamíferos que discrepa de la abundancia y composición de la fauna en general. Asimismo, el hecho de que los estudiantes tuvieran dificultades en distinguir los animales nativos de los no nativos, y que la cualidad de ser o no nativo no fuera importante respecto al afecto por ellos, dificulta que se establezca un vínculo entre la fauna y el contexto de los estudiantes. En este sentido, ya que la ausencia de esta relación cognitiva/afectiva con la fauna en particular, y con el medio ambiente en general, suele conducir a prácticas que sobrexplotan los recursos naturales y ponen en riesgo la biodiversidad, este es un problema particularmente importante para Colombia, pues en el escenario del posconflicto el Gobierno nacional espera que el ecoturismo sea el eje que permita el desarrollo sostenible de las comunidades más pobres y apartadas, las cuales, por lo general, son inmensamente ricas en recursos naturales.
Pero la ausencia de un vínculo cognitivo/ afectivo con la fauna nativa no solo tiene relevancia para la posible explotación sostenible de los recursos naturales. De hecho, la falta de conocimientos biológicos y ecológicos sobre el papel de las especies en sus respectivos ecosistemas condujo a la introducción de especies no nativas que se convirtieron en plagas. Por consiguiente, la identificación de los animales por su pertenencia a la fauna nativa o no nativa no solo tiene una gran importancia en términos biológicos y ecológicos, sino que es uno de los grandes retos en la educación ambiental sobre biodiversidad y un tema crucial que deben abordar las campañas de conservación y protección.

Todo esto evidencia la necesidad de desarrollar herramientas de educación ambiental que, además de mejorar la identificación de las especies no nativas, fortalezcan el conocimiento y el afecto por la fauna nativa, en especial por las especies menos conocidas o menos populares, como los invertebrados en general.

\section{Referencias}

All, A., Nuñez Castellar, P. E., Looy, y J. Van. (2014). Measuring effectiveness in digital game-based learning: A methodological review. International Journal of Serious Games, 1 (2), 3-21. Recuperado de http://doi. org/10.17083/ijsg.v1i2.18

Ballouard, J. M., Brischoux, F. y Bonnet, X. (2011). Children prioritize virtual exotic biodiversity over local biodiversity. PLOS ONE, 6(8), 1-8. doi.org/10.1371/journal.pone.0023152

Balmford, A., Clegg, L., Coulson, T., y Taylor, J. (2002). Why conservationists should heed Pokémon. Science (New York, N.Y.), 295(5564), 2367. doi.org/10.1126/science.295.5564.2367b 
Bednar-Friedl, B., Buijs, A., Dobrovodská, M., Dumortier, M., Eber-hard, K., Fischer, A., ... Young, J. (2009). A long-term biodiversity, ecosystem and awareness research network - results from a (pilot) survey in 8 European countries. Recuperado de https://www.cbd.int/cepa/doc/uk-macaulay-alternet.pdf

Bevilacqua, M., Ciarapica, F. E., Mazzuto, G. y Paciarotti, C. (2015). "Cook \& Teach": Learning by playing. Journal of Cleaner Production, 106, 259-271. doi. org/10.1016/i.jclepro.2014.11.085

Bierke, T., Østdahl, T., y Kleiven, J. (2003). Attitudes and activities related to urban wildlife: Pet owners and non-owners. Anthrozoos, 16(3), 252-262. doi. org/10.2752/089279303786992125

Convention on Biological Diversity. (2013). Quick guides to the Aichi Biodiversity Targets. Recuperado de http://www.cbd.int/nbsap/training/quick-guides/

Fischer, A., Langers, F., Bednar-Friedl, B., Geamana, N. y Skogen, K. (201 1). Mental representations of animal and plant species in their social contexts: Results from a survey across Europe. Journal of Environmental Psychology, 31 (2), 1 18-128. doi.org/10.1016/i.jenvp.2010.05.005

Fondo Mundial para la Naturaleza. (2016). Planeta Vivo-Informe 2016-Riesgo y resiliencia en una nueva era. Gland, Suiza. Recuperado de awsassets.panda. org/downloads/informe_planeta_vivo_2016.pdf

Fondo para el Medio Ambiente Mundial. (2009). Esfera de actividad del FMAM: Biodiversidad. Washington, DC. Recuperado de https://www.thegef.org/sites/ default/files/publications/Biodiversity-ES_2.pdf

Hodges, K. E. (2016). Enhancing student engagement and learning via the optional Biodiversity Challenge. Global Ecology and Conservation, 5, 100-107. doi. org/10.1016/i.gecco.2015.11.010

Johnson, A. (1994). Love of life - The Biophilia Hypothesis edited by Stephen R. Kellert and Edward O. Wilson. Bioscience, 44(5), 363. Recuperado de http://search. proquest.com/docview/216364190? accountid $=47900$

Kellert, S. R. (1984). Attitudes toward animals: Age-related development among children. En M. W. Fox y L. D. Mickley (eds.), Advances in Animal Welfare Science, 85, 43-60. Washington, DC. Recuperado de http://animalstudiesrepository.org/ cgi/viewcontent.cgi? article $=1001 \&$ context $=$ acwp_sata

Kellert, S. R., y Westervelt, M. O. (1983). Children's attitudes, knowledge and behaviors toward animals. Phase V. Fish and Wildlife Service (Dept. of Interior), Washington, DC.

Metrick, A. y Weitzman, M. L. (1998). Conflicts and choices in biodiversity preservation. The Journal of Economic Perspectives, 12(3), $21-34$.

Mora, F. (2017). Neuroeducación (2. ${ }^{a}$ ed). Madrid, España: Alianza. 
Moss, A. y Esson, M. (2010). Visitor interest in zoo animals and the implications for collection planning and zoo education programmes. Zoo Biology, 29(6), 715-731. doi. org/10.1002/zoo.20316

Moyer-Horner, L., Kirby, R. y Vaughan, C. (2010). Education as a tool for addressing the extinction crisis: Moving students from understanding to action. Revista de Biologia Tropical, 58(4), $1115-1126$.

Müller, E., Appleton, M. R., Valverde, A., y Reynolds, D. W. (2015). Capacity development. En G. L. Worboys, M. Lockwood, A. Kothari, S. Feary y I. Pulsford (eds.), Protected area governance and management (pp. 251 290). Canberra: ANU Press.

Navarro-Pérez, M. y Tidball, K. G. (2012). Challenges of biodiversity education: A review of education strategies for biodiversity education. International Electronic Journal of Environmental Education, 2(1), 12-30.

Otero, M. R. (1999). Psicología cognitiva, representaciones mentales e investigación en enseñanza de las ciencias. Investigações Em Ensino de Ciências, 4(2), 93-119.

Palmberg, I., Berg, I., Jeronen, E., Kärkkäinen, S., Norrgård-Sillanpää, P., Persson, C., ... Yli-Panula, E. (2015). Nordic-Baltic student teachers' identification of and interest in plant and animal species: The importance of species identification and biodiversity for sustainable development. Journal of Science Teacher Education, 26(6), 549-571. doi. org/10.1007/s10972-015-9438-z

Picard, R. W., Papert, S., Bender, W., Blumberg, B., Breazeal, C., Cavallo, D., ... Strohecker, C. (2004). Affective learning - a manifesto. BT Technology Journal, 22(4), 253-268. doi.org/10.1023/B:BTTJ.0000047603.37042.33

Prokop, P. y Tunnicliffe, S. D. (2008). "Disgusting" animals: Primary school children's attitudes and myths of bats and spiders. Eurasia Journal of Mathematics, Science and Technology Education, 4(2), 87-97.

Randler, C. (2008). Teaching species identification-A prerequisite for learning biodiversity and understanding ecology. Eurasia Journal of Mathematics, Science and Technology Education, 4(3), 223-231.

Randler, C., y Bogner, F. X. (2006). Cognitive achievements in identification skills. Journal of Biological Education, 40(4), 161 -165. doi. org/10.1080/00219266.2006.9656038

Roque De Pinho, J., Grilo, C., Boone, R. B., Galvin, K. A. y Snodgrass, J. G. (2014). Influence of aesthetic appreciation of wildlife species on attitudes towards their conservation in Kenyan agropastoralist communities. PLOS ONE, 9(2). doi.org/10.1371/journal. pone.0088842

Salas, G., Rodríguez, L. y Rivera, E. (2017). Aprendizaje basado en juegos para el cambio conductual respecto al manejo de residuos. Tecné, Episteme y Didaxis, TED, número extraordinario, , 563-571. Recuperado de http://revistas.pedagogica.edu.co/index. php/TED/article/view/4615/3795

Sauvé, L. (2004). Una cartografía de corrientes en educación ambiental. En I. Sato, Michèle, Carvalho (ed.), A pesquisa em educação ambiental: cartografias de uma identidade narrativa em formação. Porto Alegre: Artmed.

Secretaría del Convenio sobre la Diversidad Biológica. (2010). Perspectiva mundial sobre la biodiversidad 3. Montreal. Recuperado de www.cbd.int/GBO3

Sherwood, K. P., Rallis, S. F., y Stone, J. (1989). Effects of live animals vs. preserved specimens on student learning. Zoo Biology, 8(1), 99 104. doi.org/10.1002/zoo. 1430080112 
Snaddon, J. L., Turner, E. C., y Foster, W. A. (2008). Children's perceptions of rainforest biodiversity: Which Animals have the lion's share of environmental awareness? PLOS ONE, 3(7), e2579. doi.org/10.1371/journal.pone.0002579

Taratsa, A. (2010). Biodiversity in the context of environmental sustainable development. En K. Ulbrich, J. Settele y F. F. Benedict (eds.), Biodiversity in Education for Sustainable Development - Reflection on School-Research Cooperation (pp. 31 -37). Sofia: Penisoft Publishers.

Taylor, J. L. (1983). Guía sobre simulacion y juegos para la educación ambiental. Santiago de Chile: Oficina Regional de Educación de la Unesco para América Latina y el Caribe (Orelac).

Thiaw, I. y Munang, R. (2012). RIO +20 outcomes recognize the value of biodiversity and ecosystems: Implications for global, regional and national policy. Ecosystem Services, 1(1), 121-122. doi.org/10.1016/j.ecoser.2012.07.013

Tisdell, C., Wilson, C. y Swarna Nantha, H. (2006). Public choice of species for the "Ark": Phylogenetic similarity and preferred wildlife species for survival. Journal for Nature Conservation, 14(2), 97-105. doi.org/10.1016/i.jnc.2005.11.001

Turkay, S., Adinolf, S., y Tirthali, D. (2012). Collectible card games as learning tools. Procedia - Social and Behavioral Sciences, 46, 3701-3705. doi.org/10.1016/i. sbspro.2012.06.130

Ward, P. I., Mosberger, N., Kistler, C., Fischer, O., Ward, P. I., Mosberger, N., ... Fischer, O. (1998). The relationship between popularity and body size in zoo animals. Conservation Biology, 12(6), 1408-1411.

Wiley-Driscoll, J. (1995). Attitudes toward animals: Species ratings. Society and Animals, 3(2), 139-150. doi.org/10.1163/156853095X00125

Zajonc, R. B. (1980). Feeling and thinking. American Psychologist, 35(2), 151-175.

\section{Para citar este artículo}

Salas, G. (2018). Un juego como estrategia de educación ambiental sobre la biodiversidad de Colombia. Tecné, Episteme y Didaxis: TED 44, 167-184. 